\title{
Convergence Analysis of the Particle Method for the Camassa-Holm Equation*
}

\author{
Alina Chertock \\ Department of Mathematics, North Carolina State University, \\ Raleigh, NC 27695, USA. \\ E-mail: chertock@math.ncsu.edu \\ Jian-Guo Liu \\ Department of Physics and Mathematics, Duke University, \\ Duke University, Durham, NC 27708, USA. \\ E-mail: Jian-Guo.Liu@duke.edu \\ Terrance Pendleton \\ Department of Mathematics, North Carolina State University, \\ Raleigh, NC 27695, USA. \\ E-mail: tlpendle@ncsu.edu
}

\begin{abstract}
The purpose of this paper is to establish a new method for proving the convergence of the particle method applied to the Camassa-Holm $(\mathrm{CH})$ equation. The $\mathrm{CH}$ equation is a strongly nonlinear, bi-Hamiltonian, completely integrable model in the context of shallow water waves. The equation admits solutions that are nonlinear superpositions of traveling waves that have a discontinuity in the first derivative at their peaks and therefore are called peakons. This behavior admits several diverse scientific applications, but introduce difficult numerical challenges. To accurately capture these solutions, one may apply the particle method to the $\mathrm{CH}$ equation. Using the concept of space-time bounded variation, we show that the particle solution converges to a global weak solution of the $\mathrm{CH}$ equation for positive Radon measure initial data.
\end{abstract}

${ }^{*}$ The work of A. Chertock and T. Pendleton was supported in part by the NSF Grant DMS-0712898; the work of J.-G. Liu was supported in part by the NSF Grant DMS 10-11738 


\section{Introduction}

The purpose of this paper is to establish convergence results for the particle method applied to the Camassa-Holm ( $\mathrm{CH})$ equation, given as

$$
m_{t}+(u m)_{x}+u_{x} m=0 \quad \text { with } \quad m=u-\alpha^{2} u_{x x}
$$

which is considered subject to the initial condition

$$
m(x, 0)=m_{0}(x) .
$$

Here the momentum $m$ and velocity $u$ are functions of the time variable $t$ and the spatial variable $x$, and $\alpha$ is a length scale. Equation (1.1) arises in diverse scientific applications and, for instance, can be described as a bi-Hamiltonian model for waves in shallow water [3]. Equation (1.1) can also be used to quantify growth and other changes in shape, such as occurs in a beating heart, by providing the transformative mathematical path between the two shapes, (see, e.g, [8]).

The $\mathrm{CH}$ equation exhibits some remarkable properties. Of notable interest is the fact that the equation is completely integrable and yields peakon solutions which are solitons (whose identity is preserved through nonlinear interactions) with a sharp peak. Mathematically, this sharp peak is characterized by a discontinuity at the peak in the wave slope, and therefore are called peakons, [3].

Peakons may be accurately captured by applying particle methods to the $\mathrm{CH}$ equation as shown in, e.g., [4-6,8]. In these methods, the solution is sought as a linear combination of Dirac distributions, whose positions and coefficients represent locations and weights of the particles, respectively. The solution is then found by following the time evolution of the locations and the weights of these particles according to a system of ODEs obtained by considering a weak formulation of the problem. The main advantage of particle methods is their (extremely) low numerical diffusion that allows one to capture a variety of nonlinear waves with high resolution, see, e.g., [9] and references therein.

In this paper, we apply the particle method for numerical solution of the $\mathrm{CH}$ equation. We begin with a brief overview of the particle method and some of its main features which are relevant to our discussion. The main analytical results we provide is the convergence proof of the particle method. While previous convergence results have been established for this equation (e.g. see $[1,2,4,7,10]$ ), we propose a new self-contained method for showing the convergence by establishing $B V$ estimates for the particle solution. We then verify that

both the particle solution and its limit are weak solutions to the $\mathrm{CH}$ equation to complete our study on the convergence analysis.

\section{Description of a Particle Method}

In this section, we describe the particle method and how it is used to solve the CH equation. To solve (1.1), we follow [6] by searching for a weak solution as a linear combination of Dirac delta functions. The weak solution to (1.1) has the following form:

$$
\left.m^{N}(x, t)=\sum_{i=1}^{N} p_{i}(t) \delta\left(x-x_{i}(t)\right)\right) .
$$


Here, $x_{i}(t)$ and $p_{i}(t)$ represent the location of the $i$-th particle and its weight, and $N$ denotes the total number of particles. The solution is then found by solving the corresponding system of ODEs [6]:

$$
\left\{\begin{array}{l}
\frac{d x_{i}(t)}{d t}=u^{N}\left(x_{i}(t), t\right) \\
\frac{d p_{i}(t)}{d t}+u_{x}^{N}\left(x_{i}(t), t\right) p_{i}(t)=0 .
\end{array}\right.
$$

Using the special relationship between $m$ and $u$ given in (1.1), one can explicitly compute the velocity $u$ and its derivative, by the convolution $u^{N}=G * m^{N}$, where $G$ is the Green's function

$$
G(|x-y|)=\frac{1}{2 \alpha} e^{-|x-y| / \alpha}
$$

associated with the one dimensional Helmholtz operator in (1.1). Thus we have the following exact expressions for both $u(x, t)$ and $u_{x}(x, t)$ :

$$
\begin{aligned}
& u^{N}(x, t)=\frac{1}{2 \alpha} \sum_{i=1}^{N} p_{i}(t) e^{-\left|x-x_{i}(t)\right| / \alpha}, \\
& u_{x}^{N}(x, t)=-\frac{1}{2 \alpha^{2}} \sum_{i=1}^{N} p_{i}(t) \operatorname{sgn}\left(x-x_{i}(t)\right) e^{-\left|x-x_{i}(t)\right| / \alpha} .
\end{aligned}
$$

With the exception of a few isolated cases, the functions $x_{i}(t)$ and $p_{i}(t), i=1, \ldots, N$ must be determined numerically and the system (2.2) must be integrated by choosing an appropriate ODE solver. In order to start the time integration, one should choose the initial positions of particles, $x_{i}^{0}$, and the weights, $p_{i}^{0}$, so that (2.1) represents a high-order approximation to the initial data $m_{0}(x)$ in $(1.2)$, as it is shown in $[6,9]$. The latter can be done in the sense of measures on $\mathbb{R}$. Namely, we choose $\left(x_{i}(0), p_{i}(0)\right)$ in such a way such that for any test function $\phi(x) \in C_{0}^{\infty}(\mathbb{R})$, we have that

$$
\left\langle m^{N}(\cdot, 0), \phi(\cdot)\right\rangle=\int_{\mathbb{R}} m_{0}(x) \phi(x) d x \approx \sum_{i=1}^{N} p_{i}^{0} \phi\left(x_{i}\right) .
$$

where

$$
\left.m_{0}^{N}(x)=\sum_{i=1}^{N} p_{i}(0) \delta\left(x-x_{i}(0)\right)\right) .
$$

Based on (2.6), we observe that determining the initial weights, $p_{i}^{0}$, is exactly equivalent to solving a standard numerical quadrature problem.

One way of solving this problem is to first divide the computational domain $\Omega$ into $N$ nonoverlapping subdomains $\Omega_{i}$, such that their union is $\Omega$. We then set the $i$-th particle $x_{i}(0)$ to be the center of mass $\Omega_{i}$. For instance, given initial particles $\left\{x_{i}(0)\right\}_{i=1}^{N}$, we may define $\Omega_{i}$ as

$$
\Omega_{i}=\left[x_{i-1 / 2}, x_{i+1 / 2}\right]=\left\{x \mid x_{i-1 / 2} \leq x \leq x_{i+1 / 2}\right\}, \quad i=1, \ldots, N
$$


and by $x_{i}(0)=\Delta x$ the center $\Omega_{i}$. For example, a midpoint quadrature will be then given by setting $p_{i}(0)=\Delta x m_{0}\left(x_{i}(0)\right)$. In general, one can build a sequence of basis functions $\left\{\varphi_{i}(x)\right\}_{i=1}^{N}$ and approximate the initial data by taking $p_{i}(0)=\int_{\mathbb{R}} \varphi_{i}(x) d m_{0}$ in $(2.7)$. We note that the latter makes sense only if $m_{0} \in \mathcal{M}(\mathbb{R})$, where $\mathcal{M}(\mathbb{R})$ is the set of Radon measures. Furthermore, one can prove that $m_{0}^{N}$ converges weakly to $m_{0}(x)$ as $N \rightarrow \infty$.

The system (2.2) may be derived in one of two ways. Following [6], we may consider a weak formulation of the problem and make a suitable substitution to derive (2.2) or one may follow $[4,5]$ by considering the Hamiltonian structure of (1.1). Following [6], it can be shown that $x_{i}(t)$, and $p_{i}(t)$ satisfy the canonical Hamiltonian equations:

$$
\frac{d x_{i}}{d t}=\frac{\partial H^{N}}{\partial p_{i}}, \quad \frac{d p_{i}}{d t}=-\frac{\partial H^{N}}{\partial x_{i}}, \quad i=1, \ldots, N
$$

where $H^{N}(t)$ is the Hamiltonian function defined as:

$$
H^{N}(t)=\frac{1}{4 \alpha} \sum_{i=1}^{N} \sum_{i=1}^{N} p_{i}(t) p_{j}(t) e^{-\left|x_{i}(t)-x_{j}(t)\right| / \alpha},
$$

and that the total linear momentum of the particle system is conserved, that is,

$$
\frac{d}{d t}\left[\sum_{i=1}^{N} p_{i}(t)\right]=0
$$

Also, one can easily establish the following result.

Proposition 2.1. Consider the Hamiltonian function given in (2.9). Then

$$
H^{N}(t)=\frac{1}{2} \int_{-\infty}^{\infty}\left(u^{N}\right)^{2}(x, t)+\alpha^{2}\left(u_{x}^{N}\right)^{2}(x, t) d x
$$

with $u^{N}(x, t)$ and $u_{x}^{N}(x, t)$ given by (2.4) and (2.5) respectively.

Proof. From (2.4) and (2.5), we observe that

$$
\left(u^{N}\right)^{2}(x, t)=\frac{1}{4 \alpha^{2}} \sum_{i=1}^{N} \sum_{j=1}^{N} p_{i}(t) p_{j}(t) e^{-\left|x-x_{i}(t)\right| / \alpha-\left|x-x_{j}(t)\right| / \alpha}
$$

and

$$
\alpha^{2}\left(u_{x}^{N}\right)^{2}(x, t)=\frac{1}{4 \alpha^{2}} \sum_{i=1}^{N} \sum_{j=1}^{N} p_{i}(t) p_{j}(t) \operatorname{sgn}\left(x-x_{i}(t)\right) \operatorname{sgn}\left(x-x_{j}(t)\right) e^{-\left|x-x_{i}(t)\right| / \alpha-\left|x-x_{j}(t)\right| / \alpha} .
$$

Substituing (2.12) and (2.13) into (2.11), yields

$$
\begin{aligned}
& \frac{1}{2} \int_{-\infty}^{\infty}\left(u^{N}\right)^{2}(x, t)+\alpha^{2}\left(u_{x}^{N}\right)^{2}(x, t) d x= \\
& \quad=\frac{1}{8 \alpha^{2}} \sum_{i=1}^{N} \sum_{j=1}^{N} p_{i}(t) p_{j}(t) \int_{-\infty}^{\infty}\left(1+\operatorname{sgn}\left(x_{i}(t)-x\right) \operatorname{sgn}\left(x_{j}(t)-x\right)\right) e^{-\left|x-x_{i}(t)\right| / \alpha-\left|x-x_{j}(t)\right| / \alpha} d x
\end{aligned}
$$


Computing the integral in the right-hand side (RHS) of the last equation, we obtain

$$
\int_{-\infty}^{\infty}\left(1+\operatorname{sgn}\left(x_{i}(t)-x\right) \operatorname{sgn}\left(x_{j}(t)-x\right)\right) e^{-\left|x-x_{i}(t)\right| / \alpha-\left|x-x_{j}(t)\right| / \alpha} d x=2 \alpha e^{-\left|x_{i}(t)-x_{j}(t)\right| / \alpha}
$$

and thus prove the proposition.

The Hamiltonian nature of the particle system and its complete integrability allows one to establish the global existence results for the solution of (2.2) and to show that for a relatively wide class of initial data there are no particle collisions in finite times. In particular, we remark that for positive intial momenta, (2.2) has a unique global solution, and hence $p_{i}(t) \neq p_{j}(t)$ for all $i \neq j, t \geq 0$.

\section{Convergence Results}

In this section, we propose a new,concise method for showing the convergence of the particle method to a unique weak solution for the Camassa-Holm equation. It should be noted that this result has already been verfied in a varierty of ways. For instance see [6]. However, by utilizing some bounded variation estimates associated with $u^{N}$ and $u_{x}^{N}$, one may show the convergence of the particle method applied to the Camassa Holms equation by using an associated compactness result to pass the limit. As mentioned above, we will assume that the initial momenta $p_{i}(0)$ is positive and hence no two particles may cross in finite time. We begin by establishing the necessary space-time $B V$ estimates for $u_{N}$ and $u_{x}^{N}$.

\subsection{Space and Time BV Estimates}

In the following section, we recall what it means for the total variation of a function to be bounded. We then show that the total variations of $u^{N}(x, t)$ and $u_{x}^{N}(x, t)$ are bounded.

Definition 3.1. Consider a (possibly unbounded) interval $J \subseteq \mathbb{R}$ and a function $u: J \rightarrow \mathbb{R}$. The total variation of $u$ is defined as

$$
\text { Tot.Var. }\{u\} \equiv \sup \left\{\sum_{j=1}^{N}\left|u\left(x_{j}\right)-u\left(x_{j-1}\right)\right|\right\},
$$

where the supremum is taken over all $N \geq 1$ and all $(N+1)$-tuples of points $x_{j} \in J$ such that $x_{0}<x_{1}<\cdots<x_{N}$. If the right hand side of (3.1) is bounded, then we say that $u$ has bounded variation, and write $u \in B V(\mathbb{R})$.

The following theorem establishes both space and time bounded variation estimates for both $u^{N}$ and $u_{x}^{N}$.

Theorem 3.2. Let $u^{N}(x, t)$ and $u_{x}^{N}(x, t)$ be functions defined in (2.4) and (2.5), respectively. Then, both $u^{N}(x, t)$ and $u_{x}^{N}(x, t)$ are $B V$ functions in the two variables $x, t$. 
Proof. We begin with showing that Tot. Var. $\left\{u^{N}(\cdot, t)\right\}$ and Tot. Var. $\left\{u_{x}^{N}(\cdot, t)\right\}$ are bounded. Indeed, from (2.3) we have Tot.Var. $\{G(x)\}=1 / \alpha$ and Tot.Var. $\left\{G_{x}(x)\right\}=2 / \alpha^{2}$. Using the fact that the total momentum of the particle system is conserved, we obtain

$$
\begin{aligned}
& \text { Tot.Var. }\left\{u^{N}(\cdot, t)\right\} \leq \sum_{j=1}^{N} p_{j}(t) \text { Tot.Var. }\{G(x)\}=\frac{1}{\alpha} \sum_{j=1}^{N} p_{j}(t)=\frac{1}{\alpha}\left|m_{0}\right|, \\
& \text { Tot.Var. }\left\{u_{x}^{N}(\cdot, t)\right\} \leq \sum_{j=1}^{N} p_{j}(t) \text { Tot.Var. }\left\{G_{x}(x)\right\}=\frac{2}{\alpha^{2}} \sum_{j=1}^{N} p_{j}(t)=\frac{2}{\alpha^{2}}\left|m_{0}\right| .
\end{aligned}
$$

In order to prove that $u^{N}(x, t)$ and $u_{x}^{N}(x, t)$ have bounded variation with respect to $t$ as well, it now suffices to show that $u^{N}$ and $u_{x}^{N}$ are both Lipschitz continuous in time in $L^{1}$, [1, Theorem 2.6].

We first consider expression (2.4) for $u^{N}$ to have

$$
\begin{aligned}
& \int_{-\infty}^{\infty}\left|u^{N}(x, t)-u^{N}(x, s)\right| d x \\
& \quad \leq \frac{1}{2 \alpha} \int_{-\infty}^{\infty}\left|\sum_{i=1}^{N}\left(p_{i}(t) e^{-\left|x-x_{i}(t)\right| / \alpha}-p_{i}(s) e^{-\left|x-x_{i}(s)\right| / \alpha}\right)\right| d x
\end{aligned}
$$

Next, we add and subtract the term $\int_{-\infty}^{\infty} p_{i}(t) e^{-\left|x-x_{i}(s)\right|} d x$ in the last equations to obtain

$$
\begin{aligned}
\int_{-\infty}^{\infty}\left|u^{N}(x, t)-u^{N}(x, s)\right| d x & \leq \frac{1}{2 \alpha} \int_{-\infty}^{\infty} \sum_{i=1}^{N} p_{i}(t)\left|e^{-\left|x-x_{i}(t)\right| / \alpha}-e^{-\left|x-x_{i}(s)\right| / \alpha}\right| d x \\
& +\frac{1}{2 \alpha} \int_{-\infty}^{\infty} \sum_{i=1}^{N} e^{-\left|x-x_{i}(s)\right| / \alpha}\left|p_{i}(t)-p_{i}(s)\right| d x
\end{aligned}
$$

Simple calculations show that

$$
\int_{-\infty}^{\infty}\left|e^{-\left|x-x_{i}(t)\right| / \alpha}-e^{-\left|x-x_{i}(s)\right| / \alpha}\right| d x \leq 4\left|x_{i}(t)-x_{i}(s)\right| \text { and } \int_{-\infty}^{\infty} e^{-\left|x-x_{i}(t)\right| / \alpha} d x \leq 2 \alpha,
$$

and hence, we have

$$
\int_{-\infty}^{\infty}\left|u^{N}(x, t)-u^{N}(x, s)\right| d x \leq \frac{2}{\alpha} \sum_{i=1}^{N} p_{i}(t)\left|x_{i}(t)-x_{i}(s)\right|+\sum_{i=1}^{N}\left|p_{i}(t)-p_{i}(s)\right| .
$$

The sums in the RHS of (3.4) can now be estimated using the ODE system (2.2) and the facts that

$$
\begin{aligned}
\left|x_{i}(t)-x_{i}(s)\right| & =\left|\int_{s}^{t} \frac{d x_{i}}{d \tau} d \tau\right| \leq \int_{s}^{t}\left|u\left(x_{i}(\tau), \tau\right)\right| d \tau \\
& \leq \frac{1}{2 \alpha} \int_{s}^{t} \sum_{j=1}^{N} p_{j}(\tau) d \tau=\frac{1}{2 \alpha} \sum_{j=1}^{N} p_{j}(0)(t-s)=\frac{1}{2 \alpha}\left|m_{0}\right|(t-s),
\end{aligned}
$$


and

$$
\begin{aligned}
\left|p_{i}(t)-p_{i}(s)\right| & =\left|\int_{s}^{t} \frac{d p_{i}}{d \tau} d \tau\right| \leq \frac{1}{2 \alpha^{2}} \int_{s}^{t} p_{i}(\tau) \sum_{j=1}^{N} p_{j}(\tau) d \tau \\
& \leq \frac{1}{2 \alpha^{2}} \int_{s}^{t} p_{i}(\tau) d \tau \sum_{j=1}^{N} p_{j}(0)=\frac{1}{2 \alpha^{2}}\left|m_{0}\right| \int_{s}^{t} p_{i} d \tau .
\end{aligned}
$$

Also,

$$
\sum_{i=1}^{N}\left|p_{i}(t)-p_{i}(s)\right| \leq \frac{1}{2 \alpha^{2}}\left|m_{0}\right| \int_{s}^{t} \sum_{j=1}^{N} p_{i}(\tau) d \tau=\frac{1}{2 \alpha^{2}}\left|m_{0}\right|^{2}(t-s) .
$$

Substituting (3.5) and (3.6) into (3.4), yields

$$
\int_{-\infty}^{\infty}\left|u^{N}(x, t)-u^{N}(x, s)\right| d x \leq \frac{3}{2 \alpha^{2}}\left|m_{0}\right|^{2}(t-s)
$$

proving that $u^{N}$ is Lipshitz continuous in time in $L^{1}$ and thus has bounded variation with respect to both $x$ and $t$.

Similarly, for $u_{x}^{N}$ we have:

$$
\begin{aligned}
& \int_{-\infty}^{\infty}\left|u_{x}^{N}(x, t)-u_{x}^{N}(x, s)\right| d x= \\
& \quad \frac{1}{2 \alpha^{2}} \int_{-\infty}^{\infty}\left|\sum_{i=1}^{N} p_{i}(t) \operatorname{sgn}\left(x_{i}(t)-x\right) e^{-\left|x-x_{i}(t)\right| / \alpha}-p_{i}(s) \operatorname{sgn}\left(x_{i}(s)-x\right) e^{-\left|x-x_{i}(s)\right| / \alpha}\right| d x .
\end{aligned}
$$

If we add and subtract $p_{i}(s) \operatorname{sgn}\left(x_{i}(t)-x\right) e^{-\left|x-x_{i}(t)\right| / \alpha}$ in the last equation, then

$$
\begin{aligned}
& \int_{-\infty}^{\infty}\left|u_{x}^{N}(x, t)-u_{x}^{N}(x, s)\right| d x \leq \frac{1}{2 \alpha^{2}} \int_{-\infty}^{\infty} \sum_{i=1}^{N}\left|p_{i}(t)-p_{i}(s)\right| e^{-\left|x-x_{i}(t)\right| / \alpha} d x \\
& \quad+\frac{1}{2 \alpha^{2}} \sum_{i=1}^{N} p_{i}(s) \int_{-\infty}^{\infty}\left|\operatorname{sgn}\left(x_{i}(t)-x\right) e^{-\left|x-x_{i}(t)\right| / \alpha}-\operatorname{sgn}\left(x_{i}(s)-x\right) e^{-\left|x-x_{i}(t)\right| / \alpha}\right| d x \\
& \quad \leq \frac{1}{\alpha} \sum_{i=1}^{N}\left|p_{i}(t)-p_{i}(s)\right|+\frac{2}{\alpha^{2}} \sum_{i=1}^{N} p_{i}(s)\left|x_{i}(t)-x_{i}(s)\right|
\end{aligned}
$$

Substituting (3.5) and (3.6) into (3.7), we finally conclude that

$$
\int_{-\infty}^{\infty}\left|u_{x}^{N}(x, t)-u_{x}^{N}(x, s)\right| d x \leq \frac{3}{2 \alpha^{3}}\left|m_{0}\right|^{2}(t-s)
$$

which together with (3.3) proves that $u_{x}^{N}(x, t)$ is a $B V$ function in $x, t$ and the statement of the theorem. 


\subsection{Compactness and Convergence}

Now that we have established the necessary space and time bounded variation estimates for $u_{N}$ and $u_{x}^{N}$, we are now in a position to use an associated compactness property to prove convergence of the particle method. We begin this section with a definition of a weak solution to the $\mathrm{CH}$ equation. From here, one may show that the particle method applied to the $\mathrm{CH}$ equation is indeed a weak solution to (1.1). Finally, we state our main convergence result which is proved using the compactness results garnered from the $B V$ estimates established above.

Definition 3.3. $u(x, t) \in C\left(0, T ; H^{1}(\mathbb{R})\right), m(x, t)=u(x, t)-\alpha^{2} u_{x x}(x, t)$ is said to be a weak solution to (1.1) if

$$
\begin{aligned}
& \int_{-\infty}^{\infty} \phi(x, 0) m(x, 0) d x+\int_{0}^{\infty} \int_{-\infty}^{\infty}\left[\phi_{t}(x, t)-\alpha^{2} \phi_{t x x}(x, t)\right] u(x, t) d x d t \\
& \quad+\int_{0}^{\infty} \int_{-\infty}^{\infty}\left[\frac{3}{2} \phi_{x}(x, t)-\frac{\alpha^{2}}{2} \phi_{x x x}(x, t)\right] u^{2}(x, t) d x d t \\
& \quad+\int_{0}^{\infty} \int_{-\infty}^{\infty} \frac{\alpha^{2}}{2} \phi_{x}(x, t) u_{x}^{2}(x, t) d x d t=0
\end{aligned}
$$

for all $\phi \in C_{0}^{\infty}\left(\mathbb{R} \times \mathbb{R}_{+}\right)$.

Proposition 3.4. Assume that $m_{0} \in \mathcal{M}(\mathbb{R})$, then the particle solution $\left(m^{N}(x, t), u^{N}(x, t)\right)$ given by (2.1), (2.2) is a weak solution of the problem (1.1), (1.2).

Proof. Let $m^{N}(x, 0), m^{N}(x, t)$ and $u^{N}(x, t), u_{x}^{N}(x, t)$ be given by formulae (2.1) and (2.2), respectively, and $\phi \in C_{0}^{\infty}\left(\mathbb{R} \times \mathbb{R}_{+}\right)$be a test function. Then, the following relations can be easily established by direct substitutions:

$$
\begin{aligned}
& \left\langle m^{N}, \phi_{t}\right\rangle=\left\langle u^{N}, \phi_{t}-\alpha^{2} \phi_{t x x}\right\rangle, \\
& \left\langle m^{N} u^{N}, \phi_{x}\right\rangle=\left\langle\left(u^{N}\right)^{2}, \phi_{x}-\frac{\alpha^{2}}{2} \phi_{x x x}\right\rangle+\alpha^{2}\left\langle\left(u_{x}^{N}\right)^{2}, \phi_{x}\right\rangle, \\
& \left\langle m^{N} u_{x}^{N}, \phi\right\rangle=\left\langle\frac{\alpha^{2}\left(u_{x}^{N}\right)^{2}-\left(u^{N}\right)^{2}}{2}, \phi_{x}\right\rangle .
\end{aligned}
$$

Using (6.1)-(6.3) and substituting $m^{N}(x, t)$ as defined by (2.1) into (3.8), yields

$$
\begin{aligned}
& \sum_{i=1}^{N} p_{i}(0) \phi\left(x_{i}(0), 0\right)+\int_{0}^{\infty} \sum_{i=1}^{N} p_{i}(t) \phi_{t}\left(x_{i}(t), t\right) d t+ \\
& \int_{0}^{\infty} \sum_{i=1}^{N} p_{i}(t) u^{N}\left(x_{i}(t), t\right) \phi_{x}\left(x_{i}(t), t\right) d t-\int_{0}^{\infty} \sum_{i=1}^{N} p_{i}(t) u_{x}^{N}\left(x_{i}(t), t\right) \phi\left(x_{i}(t), t\right) d t=0 .
\end{aligned}
$$


We now add and subtract $\sum_{i=1}^{N} \int_{0}^{\infty} p_{i}(t) \frac{d x_{i}}{d t} \phi_{x}\left(x_{i}(t), t\right) d t$ into the last equation, use the fact that

$$
\frac{d \phi\left(x_{i}(t), t\right)}{d t}=\phi_{x}\left(x_{i}(t), t\right) \frac{d x_{i}(t)}{d t}+\phi_{t}\left(x_{i}(t), t\right)
$$

and rewrite (3.12) as follows:

$$
\begin{aligned}
& \sum_{i=1}^{N} p_{i}(0) \phi\left(x_{i}(0), 0\right)+\int_{0}^{\infty} \sum_{i=1}^{N} p_{i}(t) \frac{d \phi\left(x_{i}(t), t\right)}{d t} d t \\
& \int_{0}^{\infty} \sum_{i=1}^{N} p_{i}(t)\left[u^{N}\left(x_{i}(t), t\right)-\frac{d x_{i}(t)}{d t}\right] \phi_{x}\left(x_{i}(t), t\right) d t \\
& -\int_{0}^{\infty} \sum_{i=1}^{N} p_{i}(t) u_{x}^{N}\left(x_{i}(t), t\right) \phi\left(x_{i}(t), t\right) d t=0 .
\end{aligned}
$$

Integrating by parts the second term in the first row in (3.13), and rearranging other terms, we finally obtain:

$$
\begin{aligned}
& \int_{0}^{\infty} \sum_{i=1}^{N} p_{i}(t)\left[\frac{d x_{i}(t)}{d t}-u^{N}\left(x_{i}(t), t\right)\right] \phi_{x}\left(x_{i}(t), t\right) d t \\
& +\int_{0}^{\infty} \sum_{i=1}^{N}\left[\frac{d p_{i}(t)}{d t}+p_{i}(t) u_{x}^{N}\left(x_{i}(t), t\right)\right] \phi\left(x_{i}(t), t\right) d t=0 .
\end{aligned}
$$

Since the functions $x_{i}(t)$ and $p_{i}(t)$ satisfy the system (2.2), the last equation holds for any $\phi$ implying that $m^{N}, u^{N}$ defined by (2.1), (2.4) is a weak solution of (1.1), (1.2). This completes the proof.

We are now in a position to establish a convergence result for the particle method. Using the $B V$ estimates for $u^{N}(x, t)$ and $u_{x}^{N}(x, t)$, and the fact that the particle solution is a weak solution to the $\mathrm{CH}$ equation, we may establish the following convergence result.

Theorem 3.5. Suppose that $\left(m^{N}(x, t), u^{N}(x, t)\right)$ is a particle solution of (2.1), (2.2) with initial approximation $m^{N}(\cdot, 0) \stackrel{*}{\rightarrow} m_{0}, m_{0} \in \mathcal{M}_{+}(\mathbb{R})$. Then there exist functions $u(x, t) \in$ $B V\left(\mathbb{R} \times \mathbb{R}_{+}\right)$and $m(x, t) \in \mathcal{M}_{+}\left(\mathbb{R} \times \mathbb{R}_{+}\right)$such that $m^{N}(x, t)$ and $u^{N}(x, t)$ converge to $m(x, t)$ and $u(x, t)$, respectively in the sense of distributions as $N \rightarrow \infty$. Furthermore, the limit $(u, m)$ is the unique weak solution to the $C H$ equation (1.1) with regularity $u \in$ $C\left(0, T ; H^{1}(\mathbb{R})\right), u_{x} \in B V\left(\mathbb{R} \times \mathbb{R}_{+}\right)$.

Proof. Using $B V$ estimates for $u^{N}(x, t)$ and $u_{x}^{N}(x, t)$, we refer to the compactness property in [1, Theorem 2.4] and conclude that there exist functions $u$ and $u_{x}$ and a subsequence (still labeled as $\left.u^{N}(x, t)\right)$ such that

$$
\left\|u^{N}-u\right\|_{L_{\mathrm{loc}}^{1}\left(\mathbb{R} \times \mathbb{R}_{+}\right)} \rightarrow 0, \quad\left\|u_{x}^{N}-u_{x}\right\|_{L_{\mathrm{loc}}^{1}\left(\mathbb{R} \times \mathbb{R}_{+}\right)} \rightarrow 0
$$

as $N \rightarrow \infty$. 
From Proposition 3.4, we know that $\left(m^{N}, u^{N}\right)$ is a weak solution and thus satisfy equation (3.8). To complete the proof, we need to show that each terms in (3.8) converges to that of the limit solution $(m, u)$. Indeed, by the construction of the initial approximation, one has

$$
\lim _{N \rightarrow \infty} \int_{-\infty}^{\infty} \phi(x, 0) m^{N}(x, 0) d x=\int_{-\infty}^{\infty} \phi(x, 0) m(x, 0) d x
$$

Furthermore, for any $\phi \in C_{0}^{\infty}\left(\mathbb{R} \times \mathbb{R}_{+}\right)$, we have

$$
\begin{aligned}
& \left.\mid \iint \phi\left(u^{N}\right)^{2}-(u)^{2}\right) d x d t|=| \iint \phi\left(u^{N}+u\right)\left(u^{N}-u\right) d x d t \mid \\
& \leq\|\phi\|_{L^{\infty}}\left(\left\|u^{N}\right\|_{L^{\infty}}+\|u\|_{L^{\infty}}\right) \iint_{(x, t) \in \operatorname{supt} \text { of } \phi}\left|u^{N}(x, t)-u(x, t)\right| d x d t \rightarrow 0
\end{aligned}
$$

and thus

$$
\iint \phi\left(u^{N}\right)^{2} d x d t \rightarrow \iint \phi u^{2} d x d t
$$

as as $N \rightarrow \infty$. Similarly, we obtain

$$
\iint \phi\left(u_{x}^{N}\right)^{2} d x d t \rightarrow \iint \phi\left(u_{x}\right)^{2} d x d t
$$

This shows that the limit $(m, u)$ is indeed a weak solution to the $\mathrm{CH}$ equation.

\section{Discussion of a 2 Particle Example}

We are now in a position to solve the Camassa-Holm equation with $\alpha=1$. We first consider the two particle system of ODEs analytically, and show that the particles cannot cross in finite time. We then provide some numerical examples which illistrates some of the interesting properties about the interaction between solitons. We know from above that the $\mathrm{CH}$ equation admits peakons (solitons with a sharp peak). In mathematics and physics, a soliton is a selfreinforcing solitary wave that maintains its shape while it travels at a constant finite speed. Solitons arise as the solutions of a widespread class of weakly nonlinear dispersive partial differential equations describing physical systems. While it is usually difficult to give a precise definition of a soliton, we generally associate any solution of a nonlinear partial differential equation that

- represents a wave of permanent form

- is localized, so that it decays or approaches a constant value at infinity

- can undergo a strong interaction with other solitons and retain its identity.

as a soliton. The last property is most peculiar and is highly unusual for nonlinear partial differential equations. In our examples, we use the particle method, as well as redistribution of particles to capture this phenomenon. In particular, we consider the two particle system (i.e. a particle method for which there are only two nonzero initial particle weights) with different initial weights. 


\subsection{Positive Peakons}

Consider the corresponding system of ODEs for the two-soliton problem:

$$
\begin{aligned}
\frac{d x_{1}}{d t} & =\frac{1}{2} p_{1}(t)+\frac{1}{2} p_{2}(t) e^{-\left|x_{2}(t)-x_{1}(t)\right|} \\
\frac{d x_{2}}{d t} & =\frac{1}{2} p_{2}(t)+\frac{1}{2} p_{1}(t) e^{-\left|x_{1}(t)-x_{2}(t)\right|} \\
\frac{d p_{1}}{d t} & =\frac{1}{2} p_{1}(t) p_{2}(t) \operatorname{sgn}\left(x_{1}(t)-x_{2}(t)\right) e^{-\left|x_{1}(t)-x_{2}(t)\right|} \\
\frac{d p_{2}}{d t} & =\frac{1}{2} p_{1}(t) p_{2}(t) \operatorname{sgn}\left(x_{2}(t)-x_{1}(t)\right) e^{-\left|x_{2}(t)-x_{1}(t)\right|}
\end{aligned}
$$

To analylize the seperation of particles as $t \rightarrow \infty$, we consider the following ODE where $s(t)=x_{2}(t)-x_{1}(t)$ :

$$
\frac{d s}{d t}=\frac{1}{2}\left(p_{2}(t)-p_{1}(t)\right)\left(1-e^{-s(t)}\right)
$$

In particular, we investigate how the distance between particles change if initially, we have $x_{1}(0)<x_{2}(0)$ and $p_{1}(0)>p_{2}(0)>0$-that is, the two peakons catch up to one another eventually. Our previous analysis suggest that the peakons do get closer as $t$ gets larger, but do not cross. That is, $x_{1}(t) \neq x_{2}(t)$ in finite time. To show this analytically, we may solve the above ODE with the initial condition, $s(0)=x_{2}(0)-x_{1}(0)$, to obtain

$$
s(t)=\ln \left(1+\left(e^{s(0)}-1\right) e^{\int_{0}^{t} \frac{p_{2}(\tau)-p_{1}(\tau)}{2} d \tau}\right)
$$

From above, we observe that as long as the initial momenta is positive and if the particle weights sastify (2.2), then particles can never cross (at least analytically) because $s(t) \neq 0$ for all finite times $t$.

From above, we observe that as long as the initial momenta is positive and if the particle weights sastify (??), then particles can never cross (at least analytically) because $s(t) \neq 0$ for all finite times $t$. However, the distance between particles will move closer to each other as time increases.

We now provide some numerical examples which show how two peakons interact in different situations:

One Peakon overtakes Another: We consider the case where one peakon passes another peakon in finite time. To do so, we consider two peakons initially placed at $x_{1}(0)=0$, and $x_{2}(0)=2$ with initial weights $p_{1}(0)=2$ and $p_{2}(0)=1$. We observe that the peakon defined by its initial location at $\left(x_{1}, p_{1}\right)$ has a bigger weight and will hence move faster than the peakon defined by $\left(x_{2}, p_{2}\right)$. Thus, we expect that at some finite time, the two peakons will collide and exchange momentums. This phenomonem occurs when the machine is unable to determine the true distance between the particles. Once the particles collide, they reemerge and spread apart as time grows. 

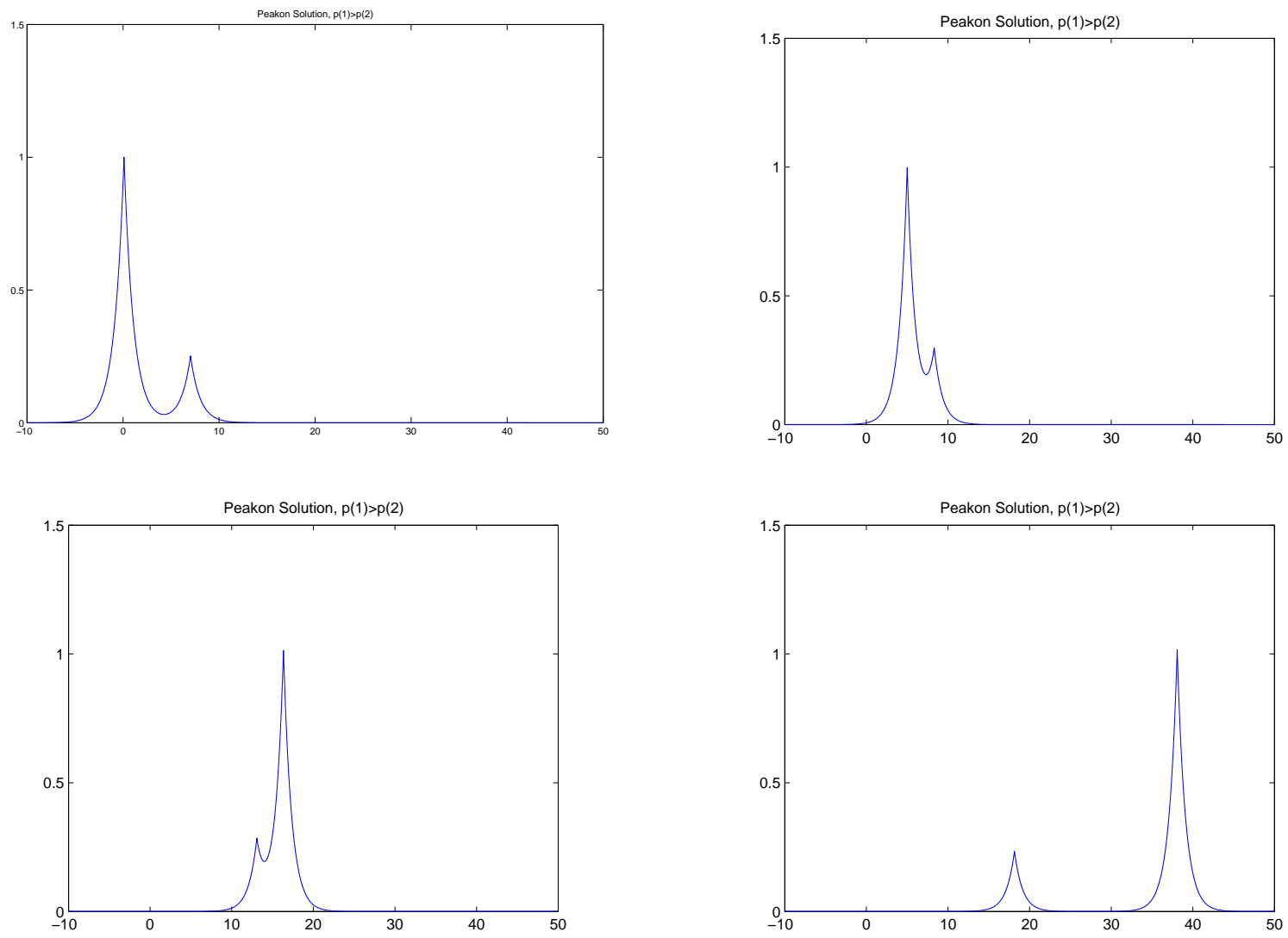

Figure 1: One Peakon Catches Up With Another

Redistribution of Particles:As mentioned in [], it may be necessary to implement a redistribution of particles to prevent the particles from artificially crossing each other. In this example, we redistribute the particles by combining their weights at a new location, once the distance between the two particles are sufficiently small. Following this method, we observe that as the particles move closer to each other, they merge into one particle whose weight approaches the sum of the two particles' individual weights. The trajectories both initially and after redistribution are given below: 

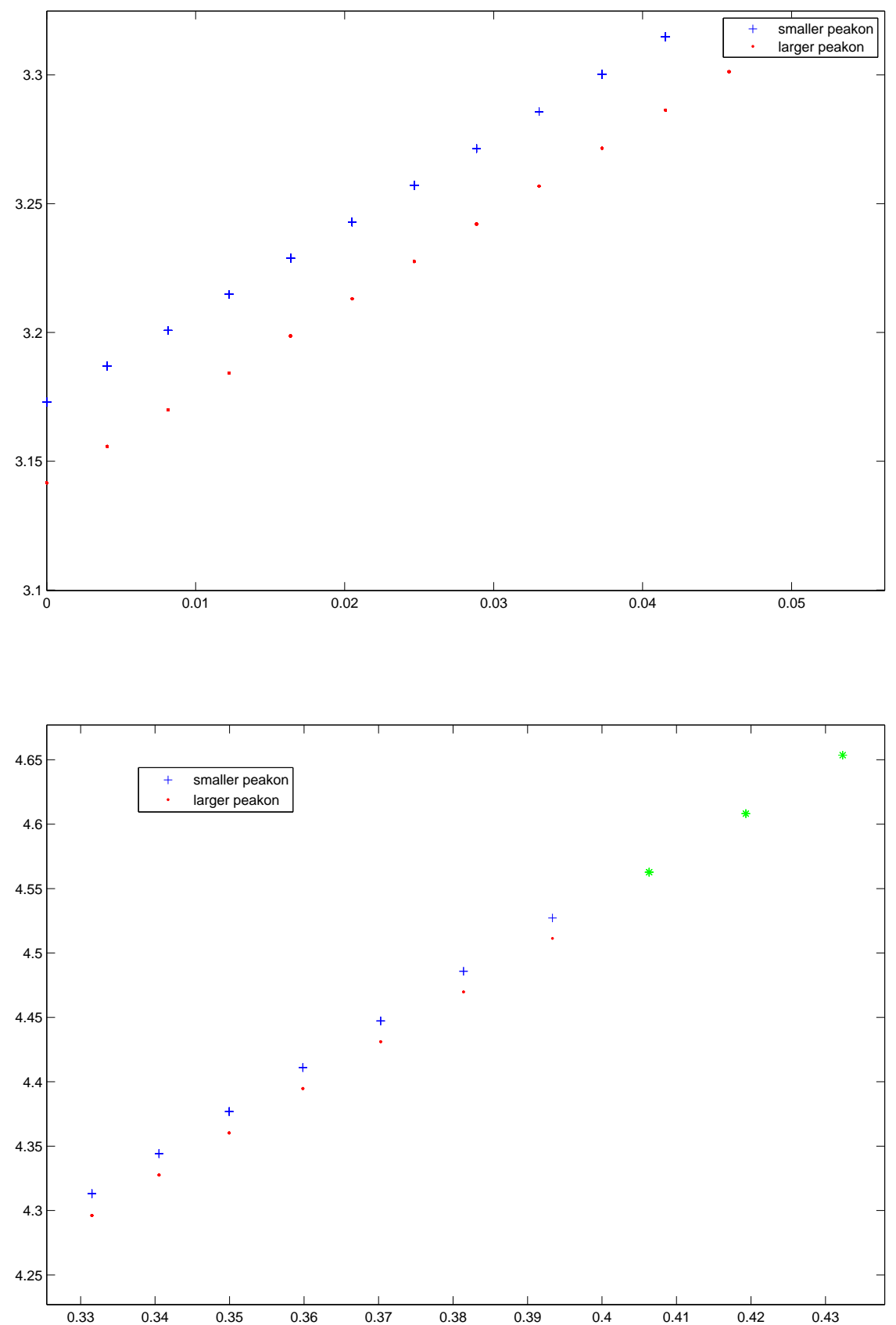

Figure 2: Redistribution of Particles

\subsection{Peakon-AntiPeakon Interaction}

If we do not require that the initial momemtum be positive, then we are no longer guaranteed that the distance between particles will be nonzero. In fact, if we consider the antipeakonpeakon solution, then we may show numerically that without redistribution, the distance 
between two nonzero particles will always be zero at some finite time. Furthermore, this particular solution is not unique. Once the peakon and antipeakon collide, we may continue the solution at $u(x, t)=0$ for all $t>t^{*}$, where $t^{*}$ is the time of collision, or we may take advantage of the symmetry of $u$, and redistribute (exchange) particles, and continue the solution. Both cases are illustrated below:
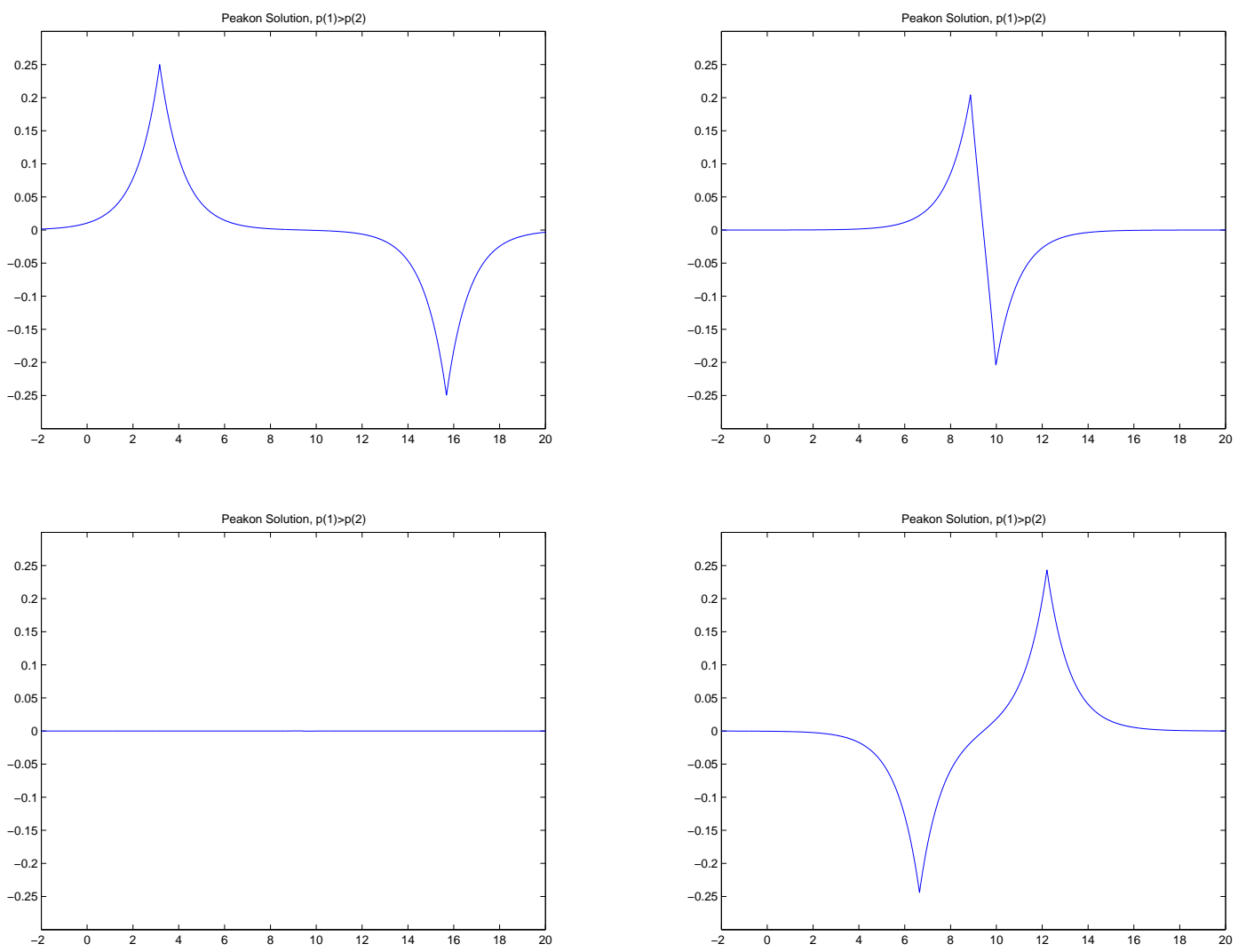

Figure 3: Peakon-AntiPeakon Solution

\section{Conclusion-Future Work}

\section{Appendix}

This appendix provides additional details and proofs of propositions omitted above.

Proposition 6.1. Suppose that $u^{N}(x, t), u_{x}^{N}(x, t)$ and $m^{N}(x, t)$ are given by (2.4), (2.5), and (2.1) respectively. Then the following relations are true for any $\phi(x, t) \in C_{0}^{\infty}\left(\mathbb{R} \times \mathbb{R}_{+}\right)$: 


$$
\begin{aligned}
& \left\langle m^{N}, \phi_{t}\right\rangle=\left\langle u^{N}, \phi_{t}-\alpha^{2} \phi_{t x x}\right\rangle \\
& \left\langle m^{N} u^{N}, \phi_{x}\right\rangle=\left\langle\left(u^{N}\right)^{2}, \phi_{x}-\frac{\alpha^{2}}{2} \phi_{x x x}\right\rangle+\alpha^{2}\left\langle\left(u_{x}^{N}\right)^{2}, \phi_{x}\right\rangle \\
& \left\langle m^{N} u_{x}^{N}, \phi\right\rangle=\left\langle\frac{\alpha^{2}\left(u_{x}^{N}\right)^{2}-\left(u^{N}\right)^{2}}{2}, \phi_{x}\right\rangle .
\end{aligned}
$$

Proof. To begin, we first show that $m^{N}(x, t)=u^{N}(x, t)-\alpha^{2} u_{x x}^{N}(x, t)$ in the sense of distributions.

Indeed for any $\phi(x, t) \in C_{0}^{\infty}\left(\mathbb{R} \times \mathbb{R}_{+}\right)$, we have the following relation by direct substitution and integration by parts:

$$
\begin{gathered}
\left\langle u^{N}-\alpha^{2} u_{x x}^{N}, \phi\right\rangle=\left\langle u^{N}, \phi\right\rangle+\alpha^{2}\left\langle u_{x}^{N}, \phi_{x}\right\rangle \\
=\frac{1}{2} \int_{0}^{\infty} \sum_{i=1}^{N} p_{i}(t) \int_{-\infty}^{\infty} e^{-\left|x-x_{i}(t)\right| / \alpha}\left(\frac{1}{\alpha} \phi(x, t)-\operatorname{sgn}\left(x-x_{i}(t)\right) \phi_{x}(x, t)\right) d x d t
\end{gathered}
$$

Now using the fact that for any $a \in \mathbb{R}$, and $\phi \in C_{0}^{\infty}(\mathbb{R})$ we have

$$
\int_{-\infty}^{a}\left(\frac{1}{\alpha} \phi(x)+\phi_{x}(x)\right) e^{-|x-a| / \alpha} d x+\int_{a}^{\infty}\left(\frac{1}{\alpha} \phi(x)-\phi_{x}(x)\right) e^{-|x-a| / \alpha} d x=2 \phi(a)
$$

we obtain that

$$
\begin{gathered}
\left\langle u^{N}, \phi\right\rangle+\alpha^{2}\left\langle u_{x}^{N}, \phi_{x}\right\rangle \\
=\int_{0}^{\infty} \sum_{i=1}^{N} p_{i}(t) \phi\left(x_{i}(t), t\right) d t \\
=\left\langle m^{N}, \phi\right\rangle
\end{gathered}
$$

From here, we see that (6.1) follows immediately via integration by parts.

Next, we verify (6.2) as follows.

Direct substitution shows that

$$
\left\langle m^{N} u^{N}, \phi_{x}\right\rangle=\frac{1}{2 \alpha} \int_{0}^{\infty} \sum_{i=1}^{N} \sum_{j=1}^{N} p_{i}(t) p_{j}(t) e^{-\left|x_{i}(t)-x_{j}(t)\right| / \alpha} \phi_{x}\left(x_{i}(t), t\right) d t
$$

Via integration by parts, we also have that

$$
\begin{gathered}
\left\langle\left(u^{N}\right)^{2}, \phi_{x}-\frac{\alpha^{2}}{2} \phi_{x x x}\right\rangle+\alpha^{2}\left\langle\left(u_{x}^{N}\right)^{2}, \phi_{x}\right\rangle=\left\langle\left(u^{N}\right)^{2}+\alpha^{2}\left(u_{x}^{N}\right)^{2}, \phi_{x}\right\rangle+\alpha^{2}\left\langle u^{N}\left(u_{x}^{N}\right), \phi_{x x}\right\rangle \\
=\int_{0}^{\infty} \int_{-\infty}^{\infty} \phi_{x}(x, t)\left[\left(\frac{1}{2 \alpha} \sum_{i=1}^{N} p_{i}(t) e^{-\left|x-x_{i}(t)\right| / \alpha}\right)^{2}+\left(\frac{1}{2 \alpha^{2}} \sum_{j=1}^{N} p_{j}(t) \operatorname{sgn}\left(x_{j}(t)-x\right) e^{-\left|x-x_{j}(t)\right| / \alpha}\right)^{2}\right] d x d t
\end{gathered}
$$


$+\int_{0}^{\infty} \int_{-\infty}^{\infty} \phi_{x x}(x, t)\left[\left(\frac{1}{2 \alpha} \sum_{i=1}^{N} p_{i}(t) e^{-\left|x-x_{i}(t)\right| / \alpha}\right)\left(\frac{1}{2 \alpha^{2}} \sum_{j=1}^{N} p_{j}(t) \operatorname{sgn}\left(x_{j}(t)-x\right) e^{-\left|x-x_{j}(t)\right| / \alpha}\right)\right] d x d t$

We consider the two-particle system and note that establishing this identity depends only on integrating with respect to $x$. Thus, it suffices to show that for $\phi \in C_{0}^{\infty}(\mathbb{R})$

$$
\begin{gathered}
\int_{-\infty}^{\infty} \phi_{x}(x)\left[\left(\frac{\gamma}{2 \alpha} e^{-|x-a| / \alpha}+\frac{\beta}{2 \alpha} e^{-|x-b| / \alpha}\right)^{2}+\left(\frac{\gamma}{2 \alpha^{2}} \operatorname{sgn}(a-x) e^{-|x-a| / \alpha}+\frac{\beta}{2 \alpha^{2}} \operatorname{sgn}(b-x) e^{-|x-b| / \alpha}\right)^{2}\right] d x \\
+\int_{-\infty}^{\infty} \phi_{x x}(x)\left[\left(\frac{\gamma}{2 \alpha} e^{-|x-a| / \alpha}+\frac{\beta}{2 \alpha} e^{-|x-b| / \alpha}\right)\left(\frac{\gamma}{2 \alpha^{2}} \operatorname{sgn}(a-x) e^{-|x-a| / \alpha}+\frac{\beta}{2 \alpha^{2}} \operatorname{sgn}(b-x) e^{-|x-b| / \alpha}\right)\right] d x \\
=\frac{\gamma \beta}{2 \alpha} e^{-|a-b| / \alpha} \phi_{x}(a)+\frac{\gamma \beta}{2 \alpha} e^{-|a-b| / \alpha} \phi_{x}(b)
\end{gathered}
$$

To show that this is indeed true we consider the case where $a<b$. We split the integral into three pieces: $x<a, a<x<b, x>b$. This reduces the integral to

$$
\int_{-\infty}^{a}\left(\phi_{x} V_{1}(x)\right)^{\prime} d x+\int_{a}^{b}\left(\phi_{x} V_{2}(x)\right)^{\prime} d x+\int_{b}^{\infty}\left(\phi_{x} V_{3}(x)\right)^{\prime} d x
$$

where

$$
\begin{aligned}
\text { - } V_{1}(x) & =\left(\frac{\gamma}{2 \alpha} e^{(x-a) / \alpha}+\frac{\beta}{2 \alpha} e^{(x-b) / \alpha}\right)^{2} \\
\text { - } V_{2}(x) & =\left(\frac{\gamma}{2 \alpha} e^{(a-x) / \alpha}+\frac{\beta}{2 \alpha} e^{(x-b) / \alpha}\right)\left(-\frac{\gamma}{2 \alpha} e^{(a-x) / \alpha}+\frac{\beta}{2 \alpha} e^{(x-b) / \alpha}\right) \\
\text { - } V_{3}(x) & =-\left(\frac{\gamma}{2 \alpha} e^{(a-x) / \alpha}+\frac{\beta}{2 \alpha} e^{(b-x) / \alpha}\right)^{2}
\end{aligned}
$$

Integrating and substituting $V_{1}, V_{2}, V_{3}$ leads us to our desired conclusion and by induction, the claim is true for $N$ particles.

Finally, we would like to show that

$$
\left\langle m^{N} u_{x}^{N}, \phi\right\rangle=\left\langle\frac{\alpha^{2}\left(u_{x}^{N}\right)^{2}-\left(u^{N}\right)^{2}}{2}, \phi_{x}\right\rangle
$$

We proceed in a similar manner as above by first observing that

$$
\left\langle m^{N} u_{x}^{N}, \phi\right\rangle=\frac{1}{2 \alpha^{2}} \int_{0}^{\infty} \sum_{i=1}^{N} \sum_{j=1}^{N} p_{i}(t) p_{j}(t) \phi\left(x_{i}(t), t\right) \operatorname{sgn}\left(x_{j}(t)-x_{i}(t)\right) e^{-\left|x_{i}(t)-x_{j}(t)\right| / \alpha} d t
$$

Now, via integration by parts, we have that

$$
\begin{gathered}
\left\langle\frac{\alpha^{2}\left(u_{x}^{N}\right)^{2}-\left(u^{N}\right)^{2}}{2}, \phi_{x}\right\rangle=\frac{1}{2}\left\langle\alpha^{2}\left(u_{x}^{N}\right)^{2}, \phi_{x}\right\rangle+\left\langle\left(u^{N}\right)\left(u_{x}^{N}\right), \phi\right\rangle \\
=\int_{0}^{\infty} \int_{-\infty}^{\infty} \phi(x, t)\left(\frac{1}{2 \alpha} \sum_{i=1}^{N} p_{i}(t) e^{-\left|x-x_{i}(t)\right| / \alpha}\right)\left(\frac{1}{2 \alpha^{2}} \sum_{j=1}^{N} p_{j}(t) \operatorname{sgn}\left(x_{j}(t)-x\right) e^{-\left|x-x_{j}(t)\right| / \alpha}\right) d x d t
\end{gathered}
$$




$$
+\frac{\alpha^{2}}{2} \int_{0}^{\infty} \int_{-\infty}^{\infty} \phi_{x}(x, t)\left(\frac{1}{2 \alpha^{2}} \sum_{j=1}^{N} p_{j}(t) \operatorname{sgn}\left(x_{j}(t)-x\right) e^{-\left|x-x_{j}(t)\right| / \alpha}\right)^{2} d x d t
$$

Once again, we consider the two-particle system and show that for any $\phi \in C_{0}^{\infty}(\mathbb{R})$,

$$
\begin{gathered}
\int_{-\infty}^{\infty} \phi(x)\left(\frac{\gamma}{2 \alpha} e^{-|x-a|}+\frac{\beta}{2 \alpha} e^{-|x-b|}\right)\left(\frac{\gamma}{2 \alpha^{2}} \operatorname{sgn}(a-x) e^{-|x-a|}+\frac{\beta}{2 \alpha^{2}} \operatorname{sgn}(b-x) e^{-|x-b|}\right) d x \\
+\frac{\alpha^{2}}{2} \int_{-\infty}^{\infty} \phi_{x}(x)\left(\frac{\gamma}{2 \alpha^{2}} \operatorname{sgn}(a-x) e^{-|x-a|}+\frac{\beta}{2 \alpha^{2}} \operatorname{sgn}(b-x) e^{-|x-b|}\right)^{2} d x \\
=\frac{1}{2 \alpha^{2}} \phi(a) \gamma \beta \operatorname{sgn}(a-b) e^{-|a-b| / \alpha}+\frac{1}{2 \alpha^{2}} \phi(b) \gamma \beta \operatorname{sgn}(b-a) e^{-|a-b| / \alpha}
\end{gathered}
$$

Similar to the previous identity, we suppose that $a<b$, and we split the integral into three intervals $(x<a, a<x<b, x>b)$. This allows us to simplify the integral to

$$
\int_{-\infty}^{a}\left(\phi W_{1}(x)\right)^{\prime} d x+\int_{a}^{b}\left(\phi W_{2}(x)\right)^{\prime} d x+\int_{b}^{\infty}\left(\phi W_{3}(x)\right)^{\prime} d x
$$

where

- $W_{1}(x)=\alpha^{2}\left(\frac{\gamma}{2 \alpha^{2}} e^{(x-a) / \alpha}+\frac{\beta}{2 \alpha^{2}} e^{(x-b) / \alpha}\right)^{2}$

- $W_{2}(x)=\frac{\alpha^{2}}{2}\left(-\frac{\gamma}{2 \alpha^{2}} e^{(a-x) / \alpha}+\frac{\beta}{2 \alpha^{2}} e^{(x-b) / \alpha}\right)^{2}$

- $W_{3}(x)=\frac{\alpha^{2}}{2}\left(-\frac{\gamma}{2 \alpha^{2}} e^{(a-x) / \alpha}-\frac{\beta}{2 \alpha^{2}} e^{(b-x) / \alpha}\right)^{2}$

Integrating and substituting $W_{1}, W_{2}$, and $W_{3}$ leads us to our desired identitity.

\section{References}

[1] A. Bressan, Hyperbolic systems of conservation laws. The one-dimensional cauchy problem, Oxford Lecture Series in Mathematics and its Applications, vol. 20, Oxford University Press, Oxford, 2000.

[2] A. Bressan and A. Constantin, Global conservative solutions of the Camassa-Holm equation, Arch. Ration. Mech. Anal. 183 (2007), no. 2, 215-239.

[3] R. Camassa and D.D. Holm, An integrable shallow water equation with peaked solitons, Phys. Rev. Lett. 71 (1993), no. 11, 1661-1664.

[4] R. Camassa, J. Huang, and L. Lee, On a completely integrable numerical scheme for a nonlinear shallow-water wave equation, J. Nonlinear Math. Phys. 12 (2005), no. 1, 146-162. 
[5] __ Integral and integrable algorithms for a nonlinear shallow-water wave equation, J. Comput. Phys. 216 (2006), no. 2, 547-572.

[6] A. Chertock, J. Marsden, and P. Du Toit, Integration of the EPDiff equation by particle methods, submitted.

[7] A. Constantin and J. Escher, Global existence and blow-up for a shallow water equation, Annali Scuola Norm. Sup. Pisa 26 (1998), 303328.

[8] D.D. Holm, T. Schmah, and C. Stoica, Geometric mechanics and symmetry, Oxford Texts in Applied and Engineering Mathematics, vol. 12, Oxford University Press, Oxford, 2009.

[9] P.-A. Raviart, An analysis of particle methods, Numerical methods in fluid dynamics (Como, 1983), Lecture Notes in Math., vol. 1127, Springer, Berlin, 1985, pp. 243-324.

[10] Z. Xin and P. Zhang, On the uniqueness and large time behavior of the weak solutions to a shallow water equation, Comm. Partial Differential Equations 27 (2002), no. 9-10, $1815-1844$. 